\title{
ESFRIs \& VO: Networking and Discussing
}

\author{
Marco Molinaro* \\ INAF - Osservatorio Astronomico di Trieste \\ via G.B. Tiepolo, 11 - 34143 Trieste, Italy \\ E-mail: marco.molinaroeinaf.it
}

\section{Fabio Pasian}

INAF - Osservatorio Astronomico di Trieste

via G.B. Tiepolo, 11 - 34143 Trieste, Italy

E-mail: fabio.pasian@inaf.it

\begin{abstract}
Open Science is the combination of Open Data and Open Access leading to interoperability of resources for science production. Fostering new science through interoperability is the goal of the International Virtual Observatory Alliance (IVOA), whose standards provide the framework to allow Open Science in Astronomy and Astrophysics.

Data Access, Discovery and Interoperability (DADI) is the ASTERICS work package dealing with the dissemination of the Virtual Observatory (VO) standards and technologies, gathering needs from the scientific community and ESFRIs requirements related to interoperability, and stirring them into updates or additions to the VO scenario.

Within DADI, networking and discussion forums and training events for the ESFRI partners (as well as for the general data providers) have been held, to put together the thinking heads of the research infrastructure (RI) projects and the staff having VO knowledge. These events initially brought the interoperability perspective to the large scientific collaborations and later allowed to feel the pulse of how much of the VO technology was welcomed, used and investigated by the RIs.

During the events, requirements and perspective on the open science scenario were brought in by the projects and collaborations and discussed with VO knowledgeable people.

This contribution tries to summarise what was discussed in such a context, in terms of how useful and practical resource interoperability is when a large collaboration has to intertwine it with project requests.
\end{abstract}

The New Era of Multi-Messenger Astrophysics - Asterics2019

25 - 29 March, 2019

Groningen, The Netherlands

\footnotetext{
*Speaker.
} 


\section{Introduction}

Within the ASTERICS project ${ }^{1}$, Work Package (WP) 4 has been dedicated to continue on the development of the Virtual Observatory (VO) framework at European level, continuing the efforts previously brought on by other EURO-VO ${ }^{2}$ projects and activities.

WP4, named $D A D I^{3}$ (Data Access, Discovery and Interoperability), described in this same volume in [1], was composed of three main tasks, one of which (Task-4.1) was meant to provide "support to astronomy ESFRI facilities, their pathfinders and other infrastructures of pan-European interest for implementation of their data in the VO framework".

In this contribution we briefly describe the activities included in the above mentioned task (Sec. 2) and then we will focus on the ESFRI Forum \& Training Event-s organised within Task-4.1 (Sec. 3), explaining the role they played in the project (Sec. 3.1) and the outcome from them (Sec. 3.2). We will then spend a few words (Sec. 4) on the integration currently starting between the VO framework and the European Open Science Cloud (EOSC ${ }^{4}$ ) initiative in terms of what it will mean for the ESFRIs and the astrophysical interoperability.

Some conclusions (Sec. 5) will summarise on the VO, Open Science and Big Data connected concepts.

\section{DADI Task-4.1}

Task-4.1 within the DADI WP of ASTERICS was meant to connect the ESFRI technical and scientific staff with the existing European VO community so that the ESFRI and pathfinder project data could be made available for discovery and usage by the whole astronomical community in an interoperable way. This support and networking task would then provide some ground for more specific technical support (Task-4.2) and gather inputs and requirements for the continuous need of update to the VO framework and standards (Task-4.3). The task's goals were reached through a set activities that included annual events and local support for training ESFRI staff.

The annual Forum and Training (F\&T) events allowed for networking, sharing knowledge, discussing requirements and providing training on how to implement data in the VO. Those events came in two flavours:

- "ESFRI Forum and Training event"(s), that we'll detail in this proceeding (Sec. 3);

- "European Data Provider Forum and Training event"(s), to enlarge the community discussion to all European Data Providers.

The two kind of events differing only in the expected audience, project restricted the first, open the the full European data providers the second (even if still keeping the focus on the ESFRI projects involved in ASTERICS). The time scaling for the events, alternating, each year, one of the two flavours, allowed for a less crowded schedule for the, usually, quite busy involved people. The

\footnotetext{
${ }^{1}$ ASTERICS: https://www.asterics2020.eu/

${ }^{2}$ EURO-VO: http://www.euro-vo.org/

${ }^{3}$ DADI: https://www.asterics2020.eu/dokuwiki/doku.php?id=open:wp4:start

${ }^{4}$ EOSC: https://ec.europa.eu/research/openscience/index.cfm?pg=open-science-cloud
} 
F\&T events themselves have been recorded as deliverables of the ASTERICS project, to keep memory of the progress, participation and discussion.

Besides these events, Task-4.1 consisted in support to ESFRI facilities to bring their data in the VO framework, through local training on existing software, Registry approach and direct visits from experienced VO staff.

\section{ESFRI Forum \& Training Events}

Held on year 1 and 3 of the ASTERICS life span, The ESFRI F\&T saw the participation of 20 to 30 people each. The first event set the common ground for discussion and brought in the preliminary requirements from the research infrastructures; the second allowed to report on the progress about the VO take-up from the ESFRIs and set the topics that future projects will have to face in the interoperability scenario.

\subsection{Role and Format}

These ESFRI dedicated events were considered the natural addition to the long standing experience with the Tech Forums within the EURO-VO community. Tech Forums have been the place for discussion and hands-on work that led to the technical progress of the VO framework in Europe (see [2] for details); the ESFRI Forums have widened the scope bringing new stakeholders in to discuss new requirements while familiarising with the VO framework.

One advantage of having these events connecting ESFRI staff with VO experts was also to help lowering the barrier, usually quite high, that even technical staff has to face when dealing for the first time with the IVOA specifications.

Not to load the usually crowded schedule of the expected participants, the meetings were organised as a couple of days packed with introductory talks and subsequent discussion. Discussion and interaction time was given high priority, leading to a roughly fifty-fifty distribution of the time span, even without taking into account additional splinter sessions (that took place also in parallel).

Attendees also were about equally distributed between VO experts and ESFRI representatives and a significant fraction of the VO expertise came directly from members of the IVOA Technical Coordination Group (TCG). The audience included mostly all partners of the DADI WP.

Before the main part of the meeting, the second event featured a dedicated session for $V O$ newcomers, to help set the basic terminology and technical blocks for ESFRI staff that were new to VO scenario. The request for such a session was a direct outcome of the previous European Data Providers F\& $\mathrm{T}^{5}$ and was useful especially for the newcomers from the EST and EGO projects. Besides proving the usefulness of having periodic forums and meetings that feed each other, this session was a precursor to similar sessions in later events at international level.

\subsection{Outcome}

The first ESFRI F\&T, apart from serving as a preliminary networking event for ESFRI staff and VO people, touched, as main topics, multidimensional data access, time domain data access, resource discovery within the Registry scenario and authentication\&authorization issues/challenges.

\footnotetext{
${ }^{5} 1^{s t}$ EDP F\&T: http://www.g-vo.org/edp-forum-2016/
} 
After two years, the topics (second ESFRI F\&T), while keeping on time domain astronomy (currently a major effort at the IVOA level), moved to radio domain data interoperability, polarimetry and spectro-polarimetry resources and reported on efforts for the provenance modelling. Also the various facets of data modelling, from representational modelling to discovery driven one, was discussed and described in its VO context.

Authentication\&authorization, or, more in general, user roles, access, storage and computation grants, still continues to be a highly discussed topic in astrophysics data and service interoperability and the two events here reported contributed to this discussion bringing together major RI requirements and perspectives.

Efforts in time domain data resource modelling, discovery and access showed a continuous effort during the evolution of the project, with requirements coming from GAIA, catalogue managers and possibly also due to those coming from LSST (not an ESFRI but nonetheless a top project in nowadays astronomical research) and its precursors.

All of this is captured in greater detail in the two project deliverable documents, D4.3 [3] and D4.10 [4], and further details can be found on the DADI wiki pages ${ }^{6,7}$ for the events on the ASTERICS web portal.

\section{EOSC integration}

ASTERICS project was a cluster project for astrophysics, including the ESFRI RIs for this research domain (even though it also included some particle physics RI). The DADI WP encompassed the activities in disseminating and evolving the $\mathrm{VO}$ technologies and approach to science openness. It did so as a WP continuing the EURO-VO projects previous efforts. In doing so it faced a larger audience than the usual IVOA European partners.

Now that ASTERICS has come to an end, continuation of the dissemination and upgrade process in interoperability moves another step forward, being these activities included within a Work Package of the recently started EU Horizon 2020 ESCAPE $^{8}$ project.

This further step has a two-fold impact. On one side ESCAPE brings together astronomy and particle physics explicitly and includes an even more larger audience. On the other side the dissemination and evolution of open science interoperability, contributed by the astronomy VO partners, is again granted one WP (WP4, CEVO, Connecting ESFRI projects to EOSC through VO framework) that, however, also includes the task to integrate the VO framework within the European Open Sciemce Cloud.

In a sense the more-than-decennial expertise on bringing astrophysical data resources to the researchers through what nowadays is called Open Science (but that has always been the natural approach in the VO data discovery and access interoperability) is facing a larger community but with less resources to cope with the requirements.

What is also requested is to bring interoperability expertise to another community (particle physics) that, even if it shares commonalities on the scientific background, has a different approach to data management and service delivery.

\footnotetext{
${ }^{6} 1^{\text {st }}$ ESFRI F\&T: https://www.asterics2020.eu/dokuwiki/doku.php?id=open:wp4:wp4esfriforum1

${ }^{7} 2^{\text {nd }}$ ESFRI F\&T: https://www.asterics2020.eu/dokuwiki/doku.php?id=open:wp4:wp4esfriforum2

${ }^{8}$ ESCAPE: https://projectescape.eu/, started Feb. 2019
} 
Finally, all of the above is done within the framework of the EOSC, an European-bound cluster approach, while the projects and collaboration involved have ongoing collaborations at global scale.

\section{Conclusions}

The ESFRI F\&T Events, held within the DADI WP of the ASTERICS project proved to be an effective way in discussing requirements from European strategic RIs in the interoperable scenario developed since 2002 by the IVOA and contributed by EURO-VO partners at continental scale.

Being VO interoperability the direct way to multi-messenger paradigm exploitation and precursor to the current Open Science efforts, this forum exercises will also be useful in the oncoming integration of activities and frameworks within the EOSC.

It's true that new challenges, in user federation, authentication and authorization, computational resources to be better connected to data resources (in a Big Data scenario) are to be faced, but it seems also true that the Virtual Observatory paradigm, despite being a worn definition, is still the right approach to science openness.

\section{Acknowledgements}

The authors acknowledge support from ASTERICS, a project funded by the European Commission under the Horizon2020 programme (grant number 653477), in the framework of ASTERICS Work Package 4 Data Access, Discover and Interoperability (DADI).

\section{References}

[1] F. Genova, M. G. Allen, C. Boisson, E. Chassande-Mottin, P. Coyle, A. Lawrence et al., Access, Discovery and Interoperability of Multi-wavelength/multi-messenger Data, in The New Era of Multi-Messenger Astrophysics - Asterics2019, vol. this volume of Proceedings of Science, 2019.

[2] F. Genova, M. G. Allen, C. Arviset, A. Lawrence, F. Pasian, E. Solano et al., Euro-VO-Coordination of virtual observatory activities in Europe, Astronomy and Computing 11 (2015) 181 [1506.06567].

[3] M. Molinaro and F. Pasian, ASTERICS GA DELIVERABLE: D4.3 - First ESFRI Forum and Training Event, tech. rep., H2020 ASTERICS, Feb., 2016.

[4] M. Molinaro and F. Pasian, ASTERICS GA DELIVERABLE: D4.10 - Second ESFRI Forum and Training Event, tech. rep., H2020 ASTERICS, Jan., 2018. 